\title{
Personalized cognitive improvement by dietary supplements
}

\begin{abstract}
Opinion
Cognition functions, including attention, memory, reasoning, and language are affected by several diseases including Alzheimer's disease, Parkinson's disease and other neurological disorders. ${ }^{1}$ Specific nootropic drugs that may improve the cognitive function in patients with memory disorders may also increase performances in healthy people. ${ }^{2}$ Moreover, natural compounds and supplements demonstrated potential adjunctive therapeutic effects on various neurodegenerative diseases. ${ }^{3}$ The global market for cognition-enhancing supplements exceeded 1 billion USD in $2015 .{ }^{4}$ Some patients are fighting agerelated cognitive decline, whereas others are focused on enhancement of their mental performance. Since the market is flooded with a plethora of cognitive-enhancing supplements, and dietary supplements are not regulated by the FDA, the effectiveness of some of these supplements may be questionable. Turmeric/curcumin and green tea extract are among the most popular dietary supplements used mainly for their anti-oxidative activities and their effect on the immune system.
\end{abstract}

\section{Turmeric/Curcumin}

Several recent clinical studies have demonstrated a positive effect of turmeric/curcumin on a variety of medical conditions. Curcumin supplementation improved artery endothelial function and reduced oxidative stress, which is associated with cardiovascular disease, in healthy middle-aged and older adults. ${ }^{5}$ Curcumin also demonstrated anti-inflammatory activity by significant reduction of serum atherosclerotic low-density lipoprotein levels in patients with mild chronic obstructive pulmonary disease (COPD). ${ }^{6}$ Curcuminoids significantly prevented the development of diabetes in a prediabetic group, ${ }^{7}$ and markedly increased anti-oxidant activity in the blood of patients with type II diabetes. ${ }^{8}$ Curcumin significantly reduced neuropathic pain, ${ }^{9}$ significantly decreased pain in aged patients with knee osteoarthritis, ${ }^{10}$ and decreased pain and prevented muscle injury after running. ${ }^{11,12}$ Supplementation of curcuminoids to standard antidepressants showed a significant reduction of anxiety and depression in patients with major depressive disorder. ${ }^{13}$ Moreover, curcumin extracts significantly improved depressive symptoms and demonstrated anxiolytic effects in patients with atypical depression. ${ }^{14}$ The positive effects on cognition was confirmed in several clinical trials. A randomized, placebo-controlled, doubleblind study demonstrated that curcumin prevented cognitive decline in older adults. ${ }^{15}$ Another study showed significant improvement of sustained attention, working memory tasks, and mood after curcumin treatment. ${ }^{16}$ Moreover, recent studies with mouse models of Alzheimer disease found that curcumin ameliorates cognitive decline and improves synaptic functions. Ongoing studies of the effect of curcumin on Alzheimer disease patients are currently being assessed. ${ }^{17}$ On the molecular level, curcumin inhibits the formation and promotes the disaggregation of amyloid- $\beta$ plaques, attenuates the hyperphosphorylation of tau and enhances its clearance, binds copper, lowers cholesterol, modifies microglial activity, inhibits acetylcholinesterase, mediates the insulin signaling pathway, and has antioxidant and anti-inflammatory activity. ${ }^{18}$
Volume II Issue I - 2018

Daniel Sliva

Purdue Research Park, USA

Correspondence: Daniel Sliva, DSTest Laboratories, Purdue Research Park, 5225 Exploration Drive Indianapolis, IN 4624I, USA, Tel 31737937 02, Email DSliva@dstest-lab.com

Received: February 21, 2018 | Published: February 28, 2018

\section{Green tea extract}

Several clinical studies found healthy effects of green tea in humans. Two independent double-blind, placebo-controlled clinical trials demonstrated that green tea extract (GTE) significantly reduced blood pressure in obese pre-hypertensive women, ${ }^{19}$ and obese hypertensive patients..$^{20}$ GTE reduced pockets of inflammation in patients with chronic periodontitis, ${ }^{21}$ and significantly reduced body weight and waist circumference in obese women. ${ }^{22}$

Neuroimaging analysis (functional magnetic resonance imaging, fMRI) of healthy volunteers showed that GTE activated the dorsolateral prefrontal cortex (DLPFC), a key area that mediates working memory in the human brain. ${ }^{23} \mathrm{~A}$ randomized, double-blind, placebo-controlled study using a combination of GTE and 1-theanine demonstrated improved memory and attention in subjects with mild cognitive impairment. ${ }^{24}$ A double-blind, placebo-controlled trial demonstrated that GTE significantly increased visual recognition memory in young adults with Down syndrome. ${ }^{25}$ Mechanistically, GTE induction of neuronal plasticity resulted in cognitive improvement. ${ }^{26}$ In addition to anti-oxidative and anti-inflammatory activities, GTE may improve cognitive function by the inhibition of arterial stiffness. ${ }^{27}$ Although the above described clinical studies demonstrated significant improvement of cognition by characterized curcumin and green tea extract, there are several concerns that need to be addressed.

i. Curcumin and green tea extracts used in clinical trials are not matching curcumin and green tea extract dietary supplements on the market. Although the producers of these supplements are declaring the chemical composition of curcumin/turmeric and green tea extracts, the amount of biologically active compounds is not identical among different producers. Since supplement effectiveness is not regulated by the FDA, consumers do not know if their supplements have any promised effects. Certainly, some of the supplements may be active, whereas others may not be active. We have recently tested and compared anti-inflammatory and anti-oxidant activity of several 
curcumin and green tea extract supplements on the US market. As expected, some of these supplements demonstrated strong activity, whereas others did not show the expected effects (Figure 1).

ii. The effectiveness of curcumin and green tea extracts in the human body depends on the bioavailability. Because of the poor bioavailability of biologically active compounds, curcuminoids in curcumin/turmeric extracts and catechins in green tea extracts, these extracts need to be modified. Several formulations were recently developed and successfully tested for improved bioavailability of curcumin and green tea extracts. ${ }^{28,29}$

iii. Although curcuminoids in curcumin/turmeric extracts and catechins in green tea extracts are not drugs, pharmacological principles apply to their efficiency. Therefore, consumers of curcumin and green tea extracts can be categorized as responders and non-responders. In other words, responders have specific set of genes, which can be regulated by curcumin and green tea extracts by genetic or epigenetic mechanisms.

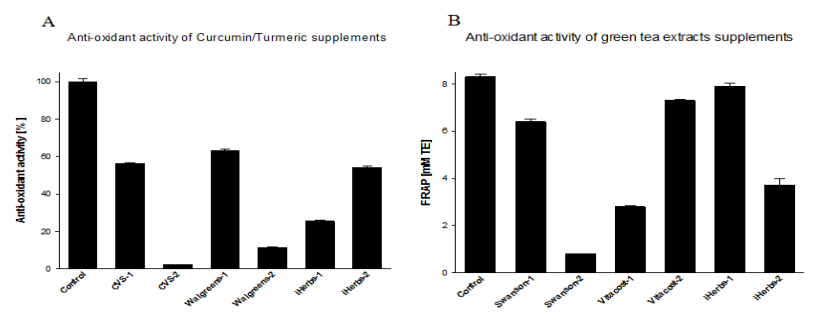

Figure I Anti-oxidant activity of Curcumin/Turmeric and green tea extract dietary supplements. (A) Anti-oxidant activity of Curcumin/ Turmeric extracts is expressed as percentage of activity compared to the same amount of control pure curcumin. (B) Anti-oxidant activity of green tea extracts is expressed as Trolox equivalent (mM TE) compared to the same amount of control pure catechin EGCG. The data are averages from three independent experiments \pm SD. Tested dietary supplements were obtained from CVS Pharmacy, Walgreens, Swanson, Vitacost and iHerbs retailers.

In summary, an improvement of cognitive functions by using dietary supplements is a complex process. Among a plethora of dietary supplements marketed for cognitive improvement, we focused on curcumin/turmeric and green tea extracts. Although clinical studies are showing promising results, a more sophisticated and personalized approach using supplements targeting specific genes is necessary. Recent international genome-wide association study (GWAS), using transcriptome-wide and epigenome-wide analysis, identified novel drug targets for several potential nootropic mechanisms [30]. Development of a novel nutrigenomic approach for targeted individual cognitive improvement by dietary supplements is the next step in the enhancement of human potential.

\section{Acknowledgment}

None.

\section{Author contributions}

All authors contributed toward data analysis, drafting and revising the paper and agree to be accountable for all aspects of the work.

\section{References}

1. Wallin A, Kettunen P, Johansson PM, et al. Cognitive medicine-a new approach in health care science. BMC Psychiatry. 2018;18(1):1-42.
2. Lanni C, Lenzken SC, Pascale A, et al. Cognition enhancers between treating and doping the mind. Pharmacol Res. 2008;57(3):196-213.

3. Bigford GE, Del Rossi G. Supplemental substances derived from foods as adjunctive therapeutic agents for treatment of neurodegenerative diseases and disorders. Adv Nutr. 2014;5(4):394-403.

4. Chinthapalli K. The billion dollar business of being smart. BMJ. (2015);351:h4829.

5. Sunagawa Y, Hirano S, Katanasaka Y, et al. Colloidal submicron-particle curcumin exhibits high absorption efficiency-a double-blind, 3-way crossover study. J Nutr Sci Vitaminol (Tokyo). 2015;61(1):37-44.

6. Funamoto M, Sunagawa Y, Katanasaka Y, et al. Highly absorptive curcumin reduces serum atherosclerotic low-density lipoprotein levels in patients with mild COPD. Int J Chron Obstruct Pulmon Dis. 2016;(11):2029-2034.

7. Chuengsamarn S, Rattanamongkolgul S, Luechapudiporn R, et al. Curcumin extract for prevention of type 2 diabetes. Diabetes Care. 2012;35(11):2121-2127.

8. Panahi Y, Khalili N, Sahebi E, et al. Antioxidant effects of curcuminoids in patients with type 2 diabetes mellitus: a randomized controlled trial. Inflammopharmacology. 2017;25(1):25-31.

9. Di Pierro F, Settembre R. Safety and efficacy of an add-on therapy with curcumin phytosome and piperine and/or lipoic acid in subjects with a diagnosis of peripheral neuropathy treated with dexibuprofen. J Pain Res. 2013;(6):497-503.

10. Panahi Y, Alishiri GH, Parvin S, et al. Mitigation of Systemic Oxidative Stress by Curcuminoids in Osteoarthritis: Results of a Randomized Controlled Trial. J Diet Suppl. 2016;13(2):209-220.

11. Nakagawa Y, Mukai S, Yamada S, et al. Short-term effects of highlybioavailable curcumin for treating knee osteoarthritis: a randomized, double-blind, placebo-controlled prospective study. J Orthop Sci. (2014);19(6):933-939.

12. Drobnic F, Riera J, Appendino G, et al. Reduction of delayed onset muscle soreness by a novel curcumin delivery system (Meriva $\left.{ }^{\circledR}\right)$ ): a randomised, placebo-controlled trial. J Int Soc Sports Nutr. (2014);11(31).

13. Panahi Y, Badeli R, Karami GR. Investigation of the efficacy of adjunctive therapy with bioavailability-boosted curcuminoids in major depressive disorder. Phytother Res. 2015;29(1):17-21.

14. Lopresti AL, Drummond PD. Efficacy of curcumin, and a saffron/curcumin combination for the treatment of major depression: A randomised, doubleblind, placebo-controlled study. J Affect Disord. 2017;(207):188-196.

15. Rainey Smith SR, Brown BM, Sohrabi HR, et al. Curcumin and cognition: a randomised, placebo-controlled, double-blind study of communitydwelling older adults. Br J Nutr. 2016;115(12):2106-2113.

16. Cox KH, Pipingas A, Scholey AB. Investigation of the effects of solid lipid curcumin on cognition and mood in a healthy older population. $J$ Psychopharmacol. 2015;29(5):642-651.

17. Reddy PH, Manczak M, Yin X, et al. Protective Effects of Indian Spice Curcumin Against Amyloid- $\beta$ in Alzheimer's Disease. $J$ Alzheimers Dis. 2018;61(3):843-866.

18. Tang M, Taghibiglou C. The Mechanisms of Action of Curcumin in Alzheimer's Disease. J Alzheimers Dis. 2017;58:1003-1016.

19. Nogueira LP, Nogueira Neto JF, Klein MR, et al. Short-term Effects of Green Tea on Blood Pressure, Endothelial Function, and Metabolic Profile in Obese Prehypertensive Women: A Crossover Randomized Clinical Trial. J Am Coll Nutr. 2017;36(2):108-115.

20. Bogdanski P, Suliburska J, Szulinska M, et al. Green tea extract reduces blood pressure, inflammatory biomarkers, and oxidative stress 
and improves parameters associated with insulin resistance in obese, hypertensive patients. Nutr Res. 2012;32(6):421-427.

21. Chava VK, Vedula BD. Thermo-reversible green tea catechin gel for local application in chronic periodontitis: a 4-week clinical trial. J Periodontol. 2013;84(9):1290-1296.

22. Chen IJ, Liu CY, Chiu JP, et al. Therapeutic effect of high-dose green tea extract on weight reduction: A randomized, double-blind, placebocontrolled clinical trial. Clin Nutr. 2013;35(3):592-599.

23. Borgwardt S, Hammann F, Scheffler K, et al. Neural effects of green tea extract on dorsolateral prefrontal cortex. Eur J Clin Nutr. (2012);66(11):1187-1192.

24. Park SK, Jung IC, Lee WK, et al. A combination of green tea extract and 1-theanine improves memory and attention in subjects with mild cognitive impairment: a double-blind placebo-controlled study. $J$ Med Food. 2011;14(4):334-343.

25. De la Torre R, de Sola S, Hernandez G, et al. Safety and efficacy of cognitive training plus epigallocatechin-3-gallate in young adults with Down's syndrome (TESDAD): a double-blind, randomised, placebocontrolled, phase 2 trial. Lancet Neurol. 2016;15(8):801-810.
26. Catuara Solarz S, Espinosa Carrasco J, Erb I, et al. Combined Treatment With Environmental Enrichment and (-)-Epigallocatechin-3-Gallate Ameliorates Learning Deficits and Hippocampal Alterations in a Mouse Model of Down Syndrome. eNeuro. 2016;3(5).

27. Li X, Lyu P, Ren Y, et al. Arterial stiffness and cognitive impairment. $J$ Neurol Sci. (2017);(380):1-10.

28. Tsuda T. Curcumin as a functional food-derived factor: degradation products, metabolites, bioactivity, and future perspectives. Food Funct. 2017;(9):705-714.

29. Rodrigues CF, Ascenção K, Silva FA, et al. Drug-delivery systems of green tea catechins for improved stability and bioavailability. Curr Med Chem. 2013;20(37):4744-4757.

30. Lam M, Trampush JW, Yu J, et al. Large-scale Cognitive GWAS MetaAnalysis Reveals Tissue-Specific Neural Expression and Potential Nootropic Drug Targets. Cell Rep. 2017;21(9):2597-2613. 